\title{
Proving HIV-1 immunity: new tools offer new opportunities
}

\author{
Louis J. Picker
}

Vaccine and Gene Therapy Institute, Oregon Health Sciences University, Mail Code: L220, 3181 SWSam Jackson Park Road, Portland, Oregon 97201-3098, USA. Phone: (503) 402-2876; Fax: (503) 402-2817; E-mail: pickerl@ohsu.edu.

In the 200 years since Jenner demonstrated that limited infection with cowpox protected from the decidedly more lethal smallpox, vaccines have been arguably the most potent weapon in the physician's arsenal. Infectious killer after infectious killer has been swept away with this approach some, like smallpox, forever. Vaccines have been so effective that belief in the potency of the immune system and the ultimate ability of vaccines to triumph over infectious disease is held almost as an article of faith by the medical profession.

The advent of HIV-1 has not shaken this basic faith, despite the fact that infection with this insidious pathogen eventually progresses in almost all individuals, many of whom manifest clear evidence of both a humoral and a cellular immune response (1). The observations that (a) the development of HIV-1-specific cytotoxic T lymphocyte (CTL) responses correlate with the drop in viral load during primary infection (2), (b) HIV-1-specific T-cell responses are generally higher in subjects with nonprogressive or slowly progressive infection (3), and (c) that HIV-1-specific T-cell responses can be detected in exposed uninfected subjects (4) have all been optimistically interpreted to reflect protective or at least effective immunity. However, our faith and optimism must be tempered with objective reasoning, and it is clear that these data have other possible interpretations. For example, given a virus that attacks the immune system, it is entirely possible that a less pathogenic viral strain might result both in no or slow disease progression, and in a quantitatively more robust immune response. Enhanced immunity in these situations may be the result, rather than the cause, of diminished infection. Similarly, an immune response might develop during an abortive infection, yet not be responsible for the failure of that infection to become established. Recent evidence in nonhuman primates supporting the effectiveness of the anti-simian immunodeficiency virus (anti-SIV) CTL responses has been quite convincing $(5,6)$, but such models may or may not accurately reflect the situation in human HIV-1 infection. There can be no substitute for direct demonstration and mechanistic characterization of "protective" immune activity in human HIV-1 infection; indeed, such data will likely be required for the successful harnessing of the immune system to combat this disease.

In this issue of the JCI, Brodie et al. (7) incorporate new analytic tools in their continuing effort to provide such direct demonstration. This group has long championed the approach of raising and expanding pathogenspecific CTL clones in vitro and then infusing large numbers of these cells back into infected subjects. This approach has been shown to be therapeutically useful for other pathogens, most notably the herpes family viruses cytomegalovirus and Epstein-Barr virus (8), and thus has the potential not only to elucidate correlates of immune protection in HIV-1 infection, but perhaps also to provide a new approach to treatment. Previous work by these investigators suggested that CTL clones can localize at sites of HIV replication and can diminish, albeit quite transiently, the numbers of circulating, productively infected $\mathrm{CD} 4^{+} \mathrm{T}$ cells (9). A major issue in this approach is the persistence and trafficking patterns of the infused effector cells; unless the cells have access to sites of viral replication, no effector response is possible. In the current manuscript (7), the authors use several quantitative approaches, including quantitative real-time PCR and PCR in situ hybridization coupled to flow cytometry, to assess persistence and trafficking of infused CTLs. They show conclusively that, while CTLs persist in the circulation for only 2 to 7 days, these cells are highly (up to 11-fold) concentrated in lymph node tissues. The authors then use sophisticated in situ PCR and immunohistochemistry to colocalize in specific areas of the lymph node activated, granzyme-positive CTL clones, resident cells with active viral replication, and cells undergoing apoptosis. Such colocalization of effector cells, infected cells, and apoptotic cells was not observed in subjects not receiving CTL infusion, suggesting that the infused CTLs were mediating an antiviral effect at the tissue level.

While these data fall short of providing definitive evidence of anti-HIV immune activity as called for above,

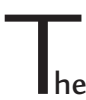

he advent of HIV-1 has not shaken

the basic faith in the potency of the immune

system or the belief in the ability of vaccines to triumph over infectious disease.

they take a critical step closer to this goal, and, most importantly, they illustrate the stepwise process by which it can most likely be accomplished. It is increasingly clear that no matter how rational the intervention, or how elegant the preclinical data supporting a given approach, complete protection is unlikely to be the outcome when any immunotherapeutic approach is first tested in the clinic. Progress will require experimental interventions, combined with analytic tools that allow the quantitative evaluation of both the cellular immune response and viral activity such that small incremental improvements can be reliably measured. Fortunately, such tools are rapidly becoming avail- 
able. In addition to the elegant quantitative real-time PCR and in situ hybridization techniques used by Brodie et al. (7), newly available techniques, such as flow cytometric analysis of antigen-stimulated intracellular cytokines and MHC-peptide tetramer staining, allow unprecedented quantification of the frequency and function of pathogen-specific $\mathrm{T}$ cells $(10-12)$. Used in conjunction with thoughtful intervention, these tools of viral and immune quantification will allow deeper understanding of the immune response and its impact on HIV and other infectious agents. The insights gained from these studies should allow us to extend the continuous chain of vaccine successes from Jenner well into the 21 st century.

1. Paul, W.E. 1995. Can the immune response control HIV infection? Cell. 82:177-182.

2. Koup, R.A., et al. 1994. Temporal association of cellular immune responses with the initial control of viremia in primary human immunodeficiency virus type 1 syndrome. J. Virol. 68:4650-4655.

3. Rosenberg, E.S., et al. 1997. Vigorous HIV-1-specific $\mathrm{CD}^{+} \mathrm{T}$ cell responses associated with control of viremia. Science. 278:1447-1450.

4. Rowland-Jones, S.L., et al. 1999. Broadly cross-reactive HIV-specific cytotoxic T-lymphocytes in highly exposed persistent seronegative donors. Immunol. Lett. 66:9-14.

5. Schmitz, J.E., et al. 1999. Control of viremia in simian immunodeficiency virus infection by CD $8+\mathrm{lym}-$ phocytes. Science. 283:857-860.

6. Evans, D.T., et al. 1999. Virus-specific cytotoxic T- lymphocyte responses select for amino-acid variation in simian immunodeficiency virus Env and Nef. Nat. Med. 5:1270-1276.

7. Brodie, S.J., et al. 2000. HIV-specific cytotoxic T lymphocytes traffic to lymph nodes and localize at sites of HIV replication and cell death.J. Clin. Invest. 105:1407-1417.

8. Riddell, S.R., and Greenberg, P.D. 1997. T cell therapy of human CMV and EBV infection in immunocompromised hosts. Rev. Med. Virol. 7:181-192.

9. Brodie, S.J., et al. 1999. In vivo migrations and function of transferred HIV-1-specific cytotoxic T cells. Nat. Med. 5:34-41.

10. Pitcher, C.J., et al. 1999. HIV-1 specific CD4+ T cells are detectable in most individuals with active HIV1 infection, but decline with prolonged viral suppression. Nat. Med. 5:518-525.

11. Kern, F., et al. 1998. T-cell epitope mapping by flow cytometry. Nat. Med. 4:975-978.

12. Ogg, G.S., et al. 1998. Quantitation of HIV-1-specific cytotoxic $\mathrm{T}$ lymphocytes and plasma load of viral RNA. Science. 279:2103-2106. 